\title{
Reconstruction Method in $F(G)$ Gravity: Stability Study and Inflationary Survey
}

\author{
C. Aïnamon1, M. J. S. Houndjo1,2, A. A. L. Ayivi', M. G. Ganiou ${ }^{3}$, A. Kanfon ${ }^{2,3}$ \\ ${ }^{1}$ Institut de Mathématiques et de Sciences Physiques (IMSP), Porto-Novo, Bénin \\ ${ }^{2}$ Faculté des Sciences et Techniques de Natitingou, Natitingou, Bénin \\ ${ }^{3}$ Département de Physique, Université d'Abomey-Calavi, Calavi, Bénin \\ Email: ainamoncyrille@yahoo.fr, sthoundjo@yahoo.fr, ayiviluc@yahoo.fr,moussiliou_ganiou @yahoo.fr, kanfon@yahoo.fr
}

How to cite this paper: Aïnamon, C., Houndjo, M.J.S., Ayivi, A.A.L., Ganiou. M.G. and Kanfon, A. (2021) Reconstruction Method in $F(G)$ Gravity: Stability Study and Inflationary Survey. Journal of Modern Physics, 12, 781-797.

https://doi.org/10.4236/jmp.2021.126050

Received: March 21, 2021

Accepted: May 10, 2021

Published: May 13, 2021

Copyright (C) 2021 by author(s) and Scientific Research Publishing Inc. This work is licensed under the Creative Commons Attribution International License (CC BY 4.0).

http://creativecommons.org/licenses/by/4.0/ Open Access

\begin{abstract}
The present paper is devoted to reconstruction and cosmological study in modified $F(G)$ theory of gravity. Our reconstruction scheme is based on Friedmann metric induced equations in modified $F(G)$ theory by supposing a power law form for the scale factor of Friedmann metric. Firstly, we deal with the stability study, the obtained model. This survey reveals that for appropriated choice of the reconstructed model parameter, this model is stable under two cosmological evolutions namely the de Sitter and the power law evolutions symbolized by the appropriated scale factor. Secondly, we investigate the inflationary survey by fitting the model with the inflation observables. These observables are determined and their comparison with Planck's results leads to a special inflationary $F(G)$ model. We prove that this model especially obtained for radiation domination evolution develops an instability, so can fall to ordinary matter domination era or dark energy domination era. This is the key of graceful exit from inflation.
\end{abstract}

\section{Keywords}

Modified Gravity, Inflation, Stability, Gauss-Bonnet

\section{Introduction}

Cosmology aims to study the universe as a whole [1]. It is based on Einstein's theory of gravity, General Relativity which has brilliantly succeeded some experiment tests. But some recent observations such as the Ia type supernova [2] [3] and the Cosmic microwave background [4] [5] impose new constraint on the current Universe content. These observations allow us to affirm that the Universe is in a phase of accelerated expansion. Thus, an alternative attempts to 
provide a plausible explanation to the present of our Universe in accordance with these observations, several theoretical approaches have emerged. These approaches can be classified into two categories [6]. The first brings together those which maintain the General Relativity as a gravitational theory and modify the content of the Universe by introducing new exotic forms of matter fields either as an inflaton field or as dark matter. The second category is made up of approaches that modify gravity theory by building theories that limit General Relativity, but with additional degrees of freedom that can lead to accelerated expansion of the Universe. The latter is called the theory of modified gravity which we quote among others: $F(R)$ theory, $F(T)$ theory, $F(R, \mathcal{T})$ theory, $F(T, \mathcal{T})$ theory, $F(G)$ theory, $F(R, G)$ theory, $F(G, \mathcal{T})$ theory where $R, T, \mathcal{T}$, denote respectively the scalar curvature, the scalar torsion and the trace of the energy-momentum tensor. Note that these modified theories of gravity have been the subject of attention of several researchers in recent years. A special modified General Relativity has recently been used for several interesting works [7] [8] [9]: the $F(G)$ theory is a modified theory of Gauss-Bonnet where $F(G)$ is a generic function of the Gauss-Bonnet invariant $G$ which is inspired by string theory and takes the form:

$$
G=R^{2}-4 R^{\mu \nu} R_{\mu v}+R^{\mu \nu \rho \sigma} R_{\mu \nu \rho \sigma}
$$

The Gauss-Bonnet term plays an important role because it makes it possible to avoid phantom contributions and helps to regulate the action of gravitation [10] [11] [12]. This 4-dimensional Gauss-Bonnet term is a topological invariant and therefore has no dynamic effect if it is added linearly to the Lagrangian. To introduce an additional dynamic, we can associate the Gauss-Bonnet term with a scalar field, as it naturally appears in the effective actions using low energy chains [13] [14]. For exponential coupling with a scalar field potential, this model can produce a period dominated by matter followed by an accelerated period [15] [16].

Several other interesting works have been done so far in this theory. The GaussBonnet scalar and the $F(G)$ theory were considered to reconstruct theories favorable to the expansion of the universe [11]. In [17], the conditions of existence and stability of cosmological solutions of the power law, when the Einstein-Hilbert action is modified by inclusion of a Gauss-Bonnet function $F(G)$, have been established. The cylindrical symmetry in $F(G)$ gravity was studied and it was shown that only three choices of $F(G)$ models are compatible with the exact solutions [18]. The same authors obtained in [19] a perfectly symmetrical and cylindrical solution in modified Gauss-Bonnet theory. A description of the deceleration-acceleration cosmological transition has been made in modified $F(G)$ and $F(R)$ theory [20] where it has been shown that a solution containing the Big-Bang and Big-Rip singularities can be reconstructed using only the auxiliary field formalism. The cosmography in modified $F(G)$ gravity theory has been studied in [21] and the authors have reconstructed the present values of $F(G)$, of its derivatives as well as those of the cosmographic parameters by 
considering a homogeneous and isotropic universe on a large scale. A cosmological study was carried out in modified Gauss-Bonnet theory [22] to account for the recent accelerated expansion of the universe. In their work, C. Bömher and F. Lobo analyzed the stability of Einstein's static universe by considering linear and homogeneous perturbations in the context of the modified GaussBonnet theory [23]; by use of a generic function $F(G)$, they showed that the region of stability of such a universe is governed by the parameter of equation of state $\omega$ and the second derivative of the model $F(G)$.

This success of the $F(G)$ theory has motivated us to take an interest in it and solving some current riddles in cosmology. The present work constitutes a contribution going in the same direction as the points previously mentioned. In addition to the benefit of being able to explain the present Universe acceleration of the by modifying standard theories of gravity, another major issue which will hopefully be explained in the next two decades, is the primordial post-quantum gravity era of our Universe. To date there are two candidate descriptions for this primordial era, the inflationary scenario [24]-[32] and the bouncing cosmology scenario [33] [34] [35]. Thus, in our present study, we will endeavor first to reconstruct a model in modified $F(G)$ gravity theory. Then, we aim to investigate the stability of the reconstructed model in order to check its possibility to render account some Universe evolution phases. Nowadays, inflation survey reveals as an excellent tool to investigate dynamical property of a cosmological model. This justifies the second part of this work where the inflationary observables will $\mathrm{b}$ addressed with the reconstructed model.

The paper is organized as follow. After providing the basic equations of the gravitational $F(G)$ theory in Section 2, we deal with the reconstruction program through the Section 3. The stability of the reconstructed model is achieved in the Section 4. The model is put at the heat of cosmological inflationary survey via the Section 5. The final section 6is devoted to the conclusion.

\section{Basic Equations of the Gravitational $F(G)$ Theory}

The action in dimension 4 of $F(G)$ gravity theory is given, as in [36] [37], by:

$$
S=\frac{1}{k} \int \sqrt{-g}\left[\frac{R}{2}+F(G)\right] \mathrm{d}^{4} x+S_{m}
$$

where $R$ is the Ricci scalar curvature, $F(G)$ is a generic function of the GaussBonnet topological invariant G, $k^{2}=8 \pi G$ et $S_{m}$ is the action of matter.

By varying this four-dimensional action with respect to the metric, we obtain the equation of the field which is written [36] [37]:

$$
\begin{gathered}
G_{\mu v}+8\left[R_{\mu \rho v \sigma}+R_{\rho \nu} g_{\sigma \mu}+\frac{1}{2}\left(g_{\mu v} g_{\sigma \rho}-g_{\mu \sigma} g_{v \rho}\right) R-R_{\rho \sigma} g_{v \mu}\right. \\
\left.-R_{\mu v} g_{\sigma \rho}+R_{\mu \sigma} g_{v \rho}\right] \nabla^{\rho} \nabla^{\sigma} F^{\prime}(G)+\left(G F^{\prime}(G)-F\right) g_{\mu v}=T_{\mu v}^{m}
\end{gathered}
$$


where $G_{\mu \nu}$ is Einstein's tensor, $T_{\mu \nu}^{m}$ the energy-momentum tensor of matter. In this work, we consider that $k^{2}=8 \pi G=1$ and the prime represents the ordinary derivative with respect to $G$. For the Robertson-Walker flat space metric which is written as:

$$
\mathrm{d} s^{2}=-\mathrm{d} t^{2}+a(t)^{2} \sum_{i}^{3} \mathrm{~d} x_{i}^{2}
$$

we have:

$$
R=6\left(\dot{H}+2 H^{2}\right) ; \quad G=24 H^{2}\left(\dot{H}+H^{2}\right)
$$

where $H$ is the Hubble parameter and the dot means () the time derivative.

Considering that the universe is flat and filled with a perfect fluid, Friedmann equations for the modified gravity $f(G)$ are written:

$$
\begin{gathered}
3 H^{2}=G F^{\prime}(G)-F-24 H^{3} \dot{F}^{\prime}(G)+\rho_{m} \\
-2 \dot{H}=-8 H^{3} \dot{F}^{\prime}(G)+16 H \dot{H} \dot{F}^{\prime}(G)+8 H^{2} \ddot{F}^{\prime}(G)+\rho_{m}
\end{gathered}
$$

The continuity equation is given by:

$$
\begin{gathered}
\dot{\rho}+3 H(\rho+P)=0 \\
\dot{\rho}+3 H \rho(1+\omega)=0
\end{gathered}
$$

where $\omega$ is the energy parameter.

The equations which constitute the basis of the theory being established, we will now design our model.

\section{Reconstruction of $F(G)$ Model}

In this section, we will reconstruct a $F(G)$ model. To do this, we choose a scale factor $a(t)$ obeying the power law and which is in the form:

$$
a(t)=a_{*}\left(\frac{t}{t_{*}}\right)^{p}
$$

where $a_{*}$ is the value that takes $a$ at the time $t_{*}$ and $p$ is a constant. In this case, the Hubble parameter satisfies the following relations:

$$
H=\frac{p}{t} ; \quad \dot{H}=-\frac{p}{t^{2}} ; \quad H^{2}=\frac{p^{2}}{t^{2}}
$$

Then, the GB invariant takes the form:

$$
G=\frac{24 p^{3}(p-1)}{t^{4}},
$$

leading to

$$
t=\left[\frac{24 p^{3}(p-1)}{G}\right]^{\frac{1}{4}}
$$

From the continuity Equation (9), we obtain: 


$$
\rho=\rho_{0}\left[\frac{24 p^{3}(p-1)}{G}\right]^{\frac{-3}{4} p(1+\omega)}
$$

Taking into account the Equations (11)-(13), the first Friedmann equation becomes:

$$
\frac{4}{p-1} G^{2} F^{\prime \prime}(G)+G F^{\prime}(G)-F(G)-\left[\frac{3 p G}{8(p-1)}\right]^{\frac{1}{2}}+\rho_{0}\left[\frac{G}{24 p^{3}(p-1)}\right]^{\frac{3}{4} p(1+\omega)}=0
$$

This equation takes the form:

$$
C G F^{\prime \prime}(G)+F^{\prime}(G)-F(G)-\sqrt{A} G^{\frac{1}{2}}+\rho_{0} \frac{G^{\alpha}}{B \alpha}=0
$$

with: $C=\frac{4}{p-1} ; \quad A=\frac{3 p}{8(p-1)} ; \quad B=24 p^{3}(p-1)$ et $\alpha=\frac{3}{4} p(1+\omega)$.

It is a differential equation in term of $\mathrm{G}$ which, after solving, gives the solution:

$$
F(G)=-\frac{4 \sqrt{A} G^{\frac{1}{2}}}{2+C}-\frac{\rho_{0} G^{\alpha}}{B^{\alpha}(-1+\alpha)(1+\alpha C)}+C_{1} G^{-\frac{1}{C}}+C_{2} G
$$

that we write more simply:

$$
F(G)=D G^{\frac{1}{2}}+E G^{\alpha}+C_{1} G^{\beta}+C_{2} G
$$

where: $D=-\frac{4 \sqrt{A}}{2+C} ; \quad E=\frac{-\rho_{0}}{B^{\alpha}(-1+\alpha)(1-\alpha C)} ; \quad \beta=-\frac{1}{C} ; C_{1}$ and $C_{2}$ being integration constants. The model (18) is a general model. The reason is that for appropriate choice of its parameters namely the parameters $C, p, \omega, \rho_{0}, C_{1}$ et $C_{2}$, the viable $F(G)$ models investigated in [9] can be recovered. Such models have the properties to describe not only cosmological evolutions leading by dark matter and the ordinary matter and also the transition between the two evolutions. To verify this expected property of the model, we will deal with its stability in the coming section.

\section{Stability of the $F(G)$ Model}

In this section, it is about the study of the stability of the $F(G)$ model constructed. To achieve this, we use de Sitter solutions and power law solutions which are techniques generally used in cosmology as shown by the work [17] [38] [39] [40] [41]. We will consider both the perturbation of matter and geometry in the general equations of motion. In the same approach with the work [42] [43], the geometric and the matter perturbations will be led respectively by the following equations

$$
\begin{array}{r}
H(t)=H_{b}(t)(1+\delta(t)), \\
\rho(t)=\rho_{b}(t)\left(1+\delta_{m}(t)\right)
\end{array}
$$


where $H_{b}(t)$ and $\rho_{b}(t)$ correspond respectively to the Hubble parameter and to the energy density of basic ordinary matter. By making an analogy with the continuity Equation (9), we can write:

$$
\dot{\rho}_{b}(t)+3 H_{b}(t) \rho_{b}(t)(1+\omega)=0
$$

whose solution is:

$$
\rho_{b}(t)=\rho_{0} \mathrm{e}^{-3(1+\omega) \int H_{b}(t) \mathrm{d} t}
$$

whith $\rho_{0}$, an intégration constant.

In order to study the linear perturbation of $H(t)$ and $\rho(t)$, we carry out a development of the model $F(G)$ in series of $G_{b}=24 H_{b}^{2}\left(\dot{H}+H_{b}^{2}\right)$ as following:

$$
F(G)=F^{b}+F_{G}^{b}\left(G-G_{b}\right)+O^{2}
$$

On the one hand, we substitute the Equations (19), (20) and (23) in Equation (6) which is the first Friedmann equation, and we obtain after simplification:

$$
\begin{aligned}
& \dot{\delta}(t)\left[24 H_{b}^{3}\left(24 H_{b}^{2} \dot{H}_{b}+24 H_{b}^{4}\right) F_{G G}^{b}-24 H_{b}^{3} F_{G}^{b}-576 H_{b}^{6}\left(48 H_{b} \dot{H}_{b}^{2}\right.\right. \\
& \left.\left.+24 H_{b}^{2} \ddot{H}_{b}+96 H_{b}^{3} \dot{H}_{b}\right) F_{G G G}^{b}\right]+\delta(t)\left[( 2 4 H _ { b } ^ { 2 } \dot { H } _ { b } + 2 4 H _ { b } ^ { 4 } ) \left(72 H_{b}^{2} \dot{H}_{b}\right.\right. \\
& \left.+96 H_{b}^{4}\right) F_{G G}^{b}-\left(72 H_{b}^{2} \dot{H}_{b}+96 H_{b}^{4}\right) F_{G}^{b}-72 H_{b}^{3}\left(48 H_{b} \dot{H}_{b}^{2}+24 H_{b}^{2} \ddot{H}_{b}\right. \\
& \left.+96 H_{b}^{3} \dot{H}_{b}\right) F_{G G}^{b}-24 H_{b}^{3}\left(48 H_{b} \dot{H}_{b}^{2}+24 H_{b}^{2} \ddot{H}_{b}+96 H_{b}^{3} \dot{H}_{b}\right)\left(72 H_{b}^{2} \dot{H}_{b}\right. \\
& \left.\left.+96 H_{b}^{4}\right) F_{G G G}^{b}-6 H_{b}^{2}\right]+\rho_{b} \delta_{m}(t)=0
\end{aligned}
$$

On the other hand, the substitution of the Equations (19) et (20) in Equation (9) leads to:

$$
\delta(t)=\frac{-\dot{\delta}_{m}(t)}{3(1+\omega) H_{b}(t)}
$$

By eliminating $\delta(t)$ from combination of Equations (24) and (25), we get the differential equation:

$$
\begin{aligned}
\frac{\mathrm{d} \delta_{m}(t)}{\delta_{m}(t)}= & 3(1+\omega) \rho_{b}\left[24 H_{b} \dot{H}_{b} F_{G}^{b}-24 H_{b} \dot{H}_{b}\left(24 H_{b}^{2} \dot{H}_{b}+24 H_{b}^{4}\right) F_{G G}^{b}\right. \\
& +576 H_{b}^{4} \dot{H}_{b}\left(48 H_{b} \dot{H}_{b}^{2}+24 H_{b}^{2} \ddot{H}_{b}+96 H_{b}^{3} \dot{H}\right) F_{G G G}^{b}+\left(24 H_{b} \dot{H}_{b}\right. \\
& \left.+24 H_{b}^{3}\right)\left(72 H_{b}^{2} \dot{H}_{b}+96 H_{b}^{4}\right) F_{G G}^{b}-\left(72 H_{b} \dot{H}_{b}+92 H_{b}^{3}\right) F_{G}^{b} \\
& -72 H_{b}^{2}\left(48 H_{b} \dot{H}_{b}^{2}+24 H_{b}^{2} \ddot{H}+96 H_{b}^{3} \dot{H}_{b}\right) F_{G G}^{b}-24 H_{b}^{2}\left(48 H_{b} \dot{H}_{b}^{2}\right. \\
& \left.\left.+24 H_{b}^{2} \ddot{H}_{b}+96 H_{b}^{3} \dot{H}_{b}\right)\left(72 H_{b}^{2} \dot{H}_{b}+96 H_{b}^{4}\right) F_{G G G}^{b}-6 H_{b}\right]^{-1} \mathrm{~d} t
\end{aligned}
$$

whose general solution is written in the form:

$$
\delta_{m}(t)=C_{0} \exp \left\{3(1+\omega) \int C_{H} \mathrm{~d} t\right\}
$$

where $C_{0}$ is an intégration constant and $C_{H}$ has for expression:

$$
\begin{aligned}
C_{H}= & \rho_{b}\left[24 H_{b} \dot{H}_{b} F_{G}^{b}-24 H_{b} \dot{H}_{b}\left(24 H_{b}^{2} \dot{H}_{b}+24 H_{b}^{4}\right) F_{G G}^{b}\right. \\
& +576 H_{b}^{4} \dot{H}_{b}\left(48 H_{b} \dot{H}_{b}^{2}+24 H_{b}^{2} \ddot{H}_{b}+96 H_{b}^{3} \dot{H}\right) F_{G G G}^{b}+\left(24 H_{b} \dot{H}_{b}\right.
\end{aligned}
$$




$$
\begin{aligned}
& \left.+24 H_{b}^{3}\right)\left(72 H_{b}^{2} \dot{H}+96 H_{b}^{4}\right) F_{G G}^{b}-\left(72 H_{b} \dot{H}_{b}+92 H_{b}^{3}\right) F_{G}^{b} \\
& -72 H_{b}^{2}\left(48 H_{b} \dot{H}_{b}^{2}+24 H_{b}^{2} \ddot{H}+96 H_{b}^{3} \dot{H}_{b}\right) F_{G G}^{b}-24 H_{b}^{2}\left(48 H_{b} \dot{H}_{b}^{2}\right. \\
& \left.\left.+24 H_{b}^{2} \ddot{H}_{b}+96 H_{b}^{3} \dot{H}_{b}\right)\left(72 H_{b}^{2} \dot{H}_{b}+96 H_{b}^{4}\right) F_{G G G}^{b}-6 H_{b}\right]^{-1}
\end{aligned}
$$

From the Equations (25) et (27), we obtain:

$$
\delta(t)=-\frac{C_{0} C_{H}}{H_{b}} \exp \left\{3(1+\omega) \int C_{H} \mathrm{~d} t\right\}
$$

In the rest of our work, we will use the stability of de Sitter solutions as well as that of power law solutions in order to appreciate the convergence of each of the perturbation terms.

\subsection{Stability of de Sitter Solutions}

De Sitter's solutions are well known in Cosmology because they constitute a perfect approximation of the exponential expansion of the Universe during its primordial inflation [44]. They are described by a constant Hubble parameter. In this context, the Hubble parameter and the associated scale factor correspond to:

$$
H_{b}(t)=H_{0} \Rightarrow a(t)=a_{0} \mathrm{e}^{H_{0} t}
$$

So the expression (22) becomes:

$$
\rho_{b}(t)=\rho_{0} \mathrm{e}^{-3(1+\omega) H_{0} t}
$$

And $C_{H}$ takes the form:

$$
\begin{aligned}
C_{H}= & \rho_{b}\left[-3 D(24)^{\frac{1}{2}} H_{0}+4 \alpha(-2+\alpha) E(24)^{\alpha} H_{0}^{4 \alpha-1}\right. \\
& \left.+4 \beta(-2+\beta) C_{1}(24)^{\beta} H_{0}^{4 \beta-1}-96 C_{2} H_{0}^{3}-6 H_{0}\right]^{-1}
\end{aligned}
$$

Moreover, from the differentiation of each member of the Equation (31), we express $\mathrm{d} t$ which allows us to write:

$$
\int C_{H} \mathrm{~d} t=-\frac{1}{3(1+\omega) H_{0}} \int \frac{C_{H}}{\rho_{b}} \mathrm{~d} \rho_{b}
$$

which give:

$$
\begin{aligned}
\int C_{H} \mathrm{~d} t= & -\frac{\rho_{b}}{3(1+\omega)}\left[-3 D(24)^{\frac{1}{2}} H_{0}^{2}+4 \alpha(-2+\alpha) E(24)^{\alpha} H_{0}^{4 \alpha}\right. \\
& \left.+4 \beta(-2+\beta) C_{1}(24)^{\beta} H_{0}^{4 \beta}-96 C_{2} H_{0}^{4}-6 H_{0}^{2}\right]^{-1}
\end{aligned}
$$

By substitution of this previous expression in the relations (27) et (29), we obtain respectively:

$$
\begin{aligned}
\delta_{m}(t)= & C_{0} \exp \left\{-\rho_{b}\left[-3 D(24)^{\frac{1}{2}} H_{0}^{2}+4 \alpha(-2+\alpha) E(24)^{\alpha} H_{0}^{4 \alpha}\right.\right. \\
& \left.\left.+4 \beta(-2+\beta) C_{1}(24)^{\beta} H_{0}^{4 \beta}-96 C_{2} H_{0}^{4}-6 H_{0}^{2}\right]^{-1}\right\}
\end{aligned}
$$


and

$$
\begin{aligned}
\delta(t)= & -C_{0} \rho_{b}\left[-3 D(24)^{\frac{1}{2}} H_{0}^{2}+4 \alpha(-2+\alpha) E(24)^{\alpha} H_{0}^{4 \alpha}\right. \\
& \left.+4 \beta(-2+\beta) C_{1}(24)^{\beta} H_{0}^{4 \beta}-96 C_{2} H_{0}^{4}-6 H_{0}^{2}\right]^{-1} \\
& \times \exp \left\{-\rho_{b}\left[-3 D(24)^{\frac{1}{2}} H_{0}^{2}+4 \alpha(-2+\alpha) E(24)^{\alpha} H_{0}^{4 \alpha}\right.\right. \\
& \left.\left.+4 \beta(-2+\beta) C_{1}(24)^{\beta} H_{0}^{4 \beta}-96 C_{2} H_{0}^{4}-6 H_{0}^{2}\right]^{-1}\right\}
\end{aligned}
$$

\subsection{Stability of Power Law Solutions}

In this study, we are interested in the solutions described by the power law of the scale factor. They are also initiated in Cosmology to describe a power law type inflation [41]. Thus, the scale factor changes according to a power of time $t$. And we have:

$$
a(t) \propto t^{n} \Rightarrow H_{b}(t)=\frac{n}{t}
$$

Note that by posing $n=p$, the scale factor of (37) corresponds to that of the Equation (10). The density of ordinary energy given by the relation (22) and the Gauss-Bonnet invariant given by (5) take the respective forms:

$$
\rho_{b}(t)=\rho_{0} t^{-3 n(1+\omega)}
$$

and

$$
G_{b}=\frac{24 n^{3}(n-1)}{t^{4}}
$$

Then the relation (28) expressing the magnitude $C_{H}$ becomes here:

$$
\begin{aligned}
C_{H}= & \rho_{0}\left\{-48 n^{2}(2 n-1)\left[\frac{1}{2} D k^{-\frac{1}{2}} g_{b}^{\frac{1+q}{4}}+\alpha E k^{\alpha-1} g_{b}^{\alpha+\frac{q-1}{4}}+\beta C_{1} k^{\beta-1} g_{b}^{\beta+\frac{q-1}{4}}\right.\right. \\
& \left.+C_{2} g_{b}^{\frac{3+q}{4}}\right]+2(24)^{2} n^{5}(n-1)(2 n-7)\left[-\frac{1}{4} D k^{-\frac{3}{2}} g_{b}^{\frac{1+q}{4}}\right. \\
& \left.+\alpha(\alpha-1) E k^{\alpha-2} g_{b}^{\alpha+\frac{q-1}{4}}+\beta(\beta-1) C_{1} k^{\beta-2} g_{b}^{\beta+\frac{q-1}{4}}\right] \\
& +8(24)^{3} n^{8}(n-1)(2 n-1)\left[\frac{3}{8} D k^{-\frac{5}{2}} g_{b}^{\frac{1+q}{4}}+\alpha(\alpha-1)(\alpha-2) E k^{\alpha-3} g_{b}^{\alpha+\frac{q-1}{4}}\right. \\
& \left.\left.+\beta(\beta-1)(\beta-2) C_{1} k^{\beta-3} g_{b}^{\beta+\frac{q-1}{4}}\right]-6 n g_{b}^{\frac{1+q}{4}}\right\}^{-1}
\end{aligned}
$$

avec $q=-3 n(1+\omega) ; k=24 n^{3}(n-1)$ et $g_{b}(t)=\frac{G_{b}(t)}{k}$

Taking into account the Equation (39), we deduce: $\mathrm{d} t=-\frac{1}{4} g_{b}^{-\frac{5}{4}} \mathrm{~d} g_{b}$. 
It follows:

$$
\begin{aligned}
\int C_{H} \mathrm{~d} t= & -\frac{\rho_{0}}{4} \int\left\{-48 n^{2}(2 n-1)\left[\frac{1}{2} D k^{-\frac{1}{2}} g_{b}^{\frac{q+6}{4}}+\alpha E k^{\alpha-1} g_{b}^{\alpha+\frac{q+4}{4}}\right.\right. \\
& \left.+\beta C_{1} k^{\beta-1} g_{b}^{\beta+\frac{q+4}{4}}+C_{2} g_{b}^{\frac{q+8}{4}}\right]+2(24)^{2} n^{5}(n-1)(2 n-7)\left[-\frac{1}{4} D k^{-\frac{3}{2}} g_{b}^{\frac{q+6}{4}}\right. \\
& \left.+\alpha(\alpha-1) E k^{\alpha-2} g_{b}^{\alpha+\frac{q+4}{4}}+\beta(\beta-1) C_{1} k^{\beta-2} g_{b}^{\beta+\frac{q+4}{4}}\right] \\
& +8(24)^{3} n^{8}(n-1)(2 n-1)\left[\frac{3}{8} D k^{-\frac{5}{2}} g_{b}^{\frac{q+6}{4}}+\alpha(\alpha-1)(\alpha-2) E k^{\alpha-3} g_{b}^{\alpha+\frac{q+4}{4}}\right. \\
& \left.\left.+\beta(\beta-1)(\beta-2) C_{1} k^{\beta-3} g_{b}^{\beta+\frac{q+4}{4}}\right]-6 n g_{b}^{\frac{q+6}{4}}\right\}^{-1} \mathrm{~d} g_{b}
\end{aligned}
$$

Note that we have not found an analytical solution for the integral of the relation (41), then a numerical study will be carried out in order to obtain the perturbation functions and to discuss their convergence.

\subsection{Stability Analysis}

In this part of the work, we will deal with the evolution survey of the solutions obtained as a time function. We emphasize here that in the case of the study of de Sitter's solutions, an explicit analytical expression of each of the perturbation functions $\delta_{m}(t)$ and $\delta(t)$ is obtained respectively in (35) and (36) respectively. And we can easily notice that for an appropriate choice of parameters, the quantities $\delta_{m}(t)$ and $\delta(t)$ converge. Indeed, when $t$ straights forward to infinity, the fonction $\rho_{b}(t)=\rho_{0} \mathrm{e}^{-3(1+\omega) H_{0} t}$ tends to 0 for positive or zero $\omega$. Then, for $\rho_{b}(t)$ tending to 0 , we see that $\delta_{m}(t)$ tends to $C_{0}$ (which is an arbitrary constant) and $\delta(t)$ tends to 0 . Hence these two functions converge. In the case the power law solutions, the idea was to calculate the integral given by the Equation (41) then introduce the result in each of the Equations (27) and (29) in order to obtain the respective expressions of $\delta_{m}(t)$ and $\delta(t)$. But, it turns out that the analytical solution of the Equation (41) is not easy to get, so we proceeded to a numerical study. Thus, our approach at this level consists to plot the perturbation functions $\delta_{m}(t)$ and $\delta(t)$ without having obtained their analytical expressions; which can permit us to appreciate their convergence. These curves are those of Figure 1 and Figure 2 below. They show the behaviors of $\delta_{m}(t)$ and $\delta(t)$ respectively for a Universe dominated by ordinary matter $(\omega=0)$ and for a Universe dominated by radiation $(\omega=1 / 3)$. We also note that these disturbance functions are convergent.

The convergence of these quantities for different values of the parameters of the reconstructed model, according to the de Sitter approach and the power law approach, proves that this model is stable. It is therefore conducive to the reproduction of the stage dominated by matter, radiation and also the phase characterized by de Sitter inflation [42]. 

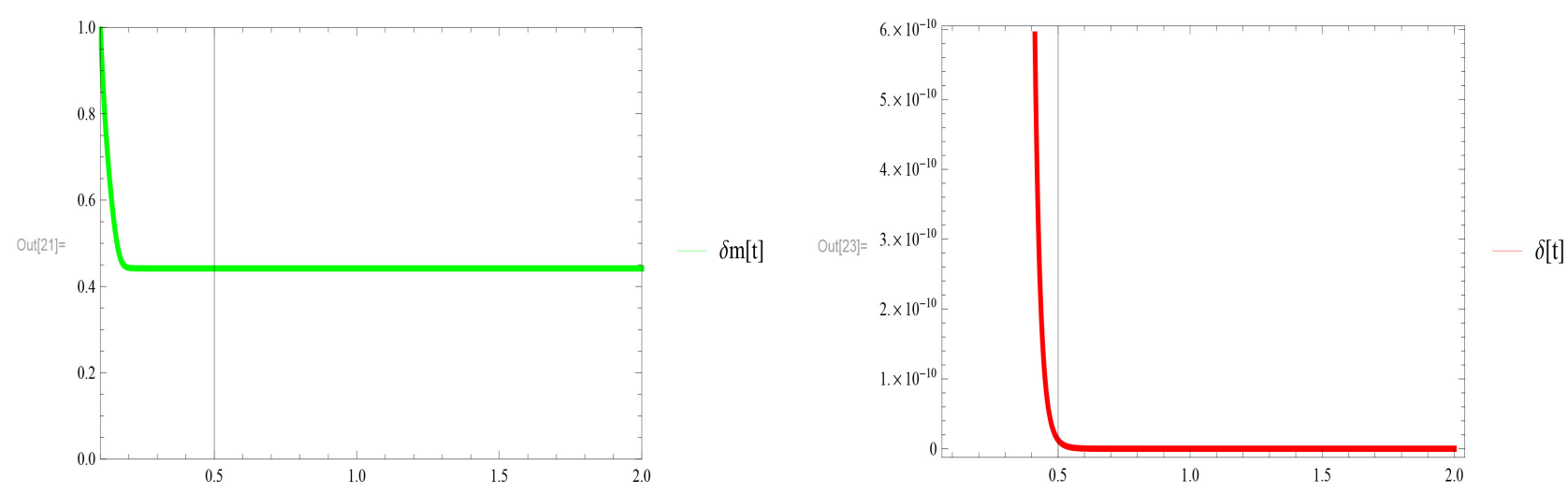

Figure 1. The perturbation functions of matter (green curve) and geometry (red curve), for: $\omega=0, n=8 ; \rho_{0}=10^{-9} ; C_{1}=30$ and $C_{2}=100$.
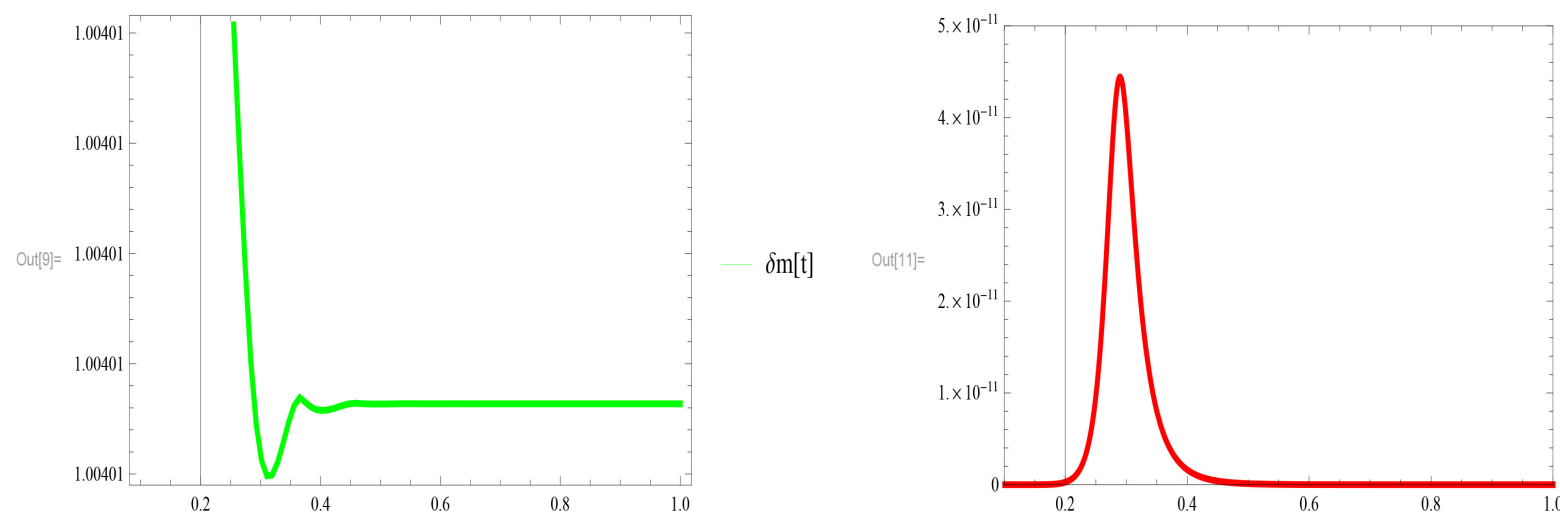

Figure 2. The perturbation functions of matter (green curve) and geometry (red curve), for: $\omega=1 / 3, n=8 ; \rho_{0}=10^{-9} ; C_{1}=30$ and $C_{2}=100$.

\section{Cosmological Inflation}

In this section of our work, we study cosmological inflation. In particular, we will determine the values of the observables of the inflation phenomenon within the framework of a scale factor obeying the power law. Taking into consideration the Planck's results, we will deduce a family of models $f(G)$ capable of describing inflation.

\subsection{Slow-Roll Parameters and Inflationary Observables}

In cosmological inflation survey, several works have always been based on the study of the inflationary observables [38]. These are the scalar spectral index of the curvature perturbations $n_{s}$, the running $\alpha_{s} \equiv \frac{\mathrm{d} n_{s}}{\mathrm{~d} \ln k}$ of the spectral index $n_{s}$ where $k$ is the absolute value of the wave number $k$, the spectral index $n_{T}$ of the tensor and the ratio $r$.

From the scalar potential denoted $V(\phi)$ characterizing inflation and its derivatives, the parameters of the slow-roll are defined as follows [39] [40]: 


$$
\begin{aligned}
\varepsilon & \equiv \frac{M_{p}^{2}}{2}\left(\frac{1}{V} \frac{\mathrm{d} V}{\mathrm{~d} \phi}\right)^{2} \\
\eta & \equiv \frac{M_{p}^{2}}{2} \frac{\mathrm{d}^{2} V}{\mathrm{~d} \phi^{2}} \\
\xi^{2} & \equiv \frac{M_{p}^{4}}{V^{2}} \frac{\mathrm{d} V}{\mathrm{~d} \phi} \frac{\mathrm{d}^{3} V}{\mathrm{~d} \phi^{3}}
\end{aligned}
$$

Note that the inflation ends when $\varepsilon=1$.

We have the approximate expressions of the inflation observables as a function of the slow-roll parameters relative to the potential. They are written according to [39] [40]:

$$
\begin{gathered}
r \approx 16 \varepsilon \\
n_{s} \approx 1-16 \varepsilon+2 \eta \\
\alpha_{s} \approx 16 \varepsilon \eta-24 \varepsilon^{2}-2 \xi^{2} \\
n_{T} \approx 16-2 \varepsilon
\end{gathered}
$$

Let us specify that in modified theory of gravity, it is not possible to exploit the conformal transformation of the Einstein theory because one cannot define neither a scalar potential, nor the parameters of the solw-rool relating to it. We then introduce the Hubble slow-roll parameters $\varepsilon_{n}$ which are defined by:

$$
\varepsilon_{n+1} \equiv \frac{\mathrm{d} \ln \left|\varepsilon_{n}\right|}{\mathrm{d} N}
$$

with $\varepsilon_{0} \equiv H_{i n i} / H$ and $N \equiv \ln \left(a / a_{i n i}\right)$ the e-folding number and where $a_{i n i}$ is the scale factor at the start of inflation and $H_{i n i}$ is the corresponding Hubble parameter. It follows:

$$
\begin{gathered}
\varepsilon_{1} \equiv \frac{\dot{H}}{H^{2}} \\
\varepsilon_{2} \equiv \frac{\ddot{H}}{\dot{H} H}-\frac{2 \dot{H}}{H^{2}} \\
\varepsilon_{3} \equiv\left(\ddot{H} H-2 \dot{H}^{2}\right)^{-1}\left[\frac{\dddot{H} \dot{H} H-\ddot{H}\left(\dot{H}^{2}+\ddot{H} H\right)}{\dot{H} H}-\frac{2 \dot{H}}{H^{2}}\left(\ddot{H} H-2 \dot{H}^{2}\right)\right]
\end{gathered}
$$

Thus, we obtain as in [39] [40] the inflation observables which are written:

$$
\begin{gathered}
r \approx 16 \varepsilon_{1} \\
n_{s} \approx 1-2 \varepsilon_{1}-2 \varepsilon_{2} \\
\alpha_{s} \approx-2 \varepsilon_{1} \varepsilon_{2}-\varepsilon^{2} \varepsilon_{3} \\
n_{T} \approx-2 \varepsilon_{1}
\end{gathered}
$$

These observables thus expressed can be easily determined. Particularly within the framework of our study where we considered a scale factor evolving according to the power law. 


\subsection{Inflationary $F(G)$ Model in Agreement with Planck's Results}

Recall that our model (18) was reconstructed from the first Friedmann equation. By ejecting this model into the second Friedmann equation, we get the following differential equation

$$
\begin{aligned}
& 2 \dot{H}(1+\omega)\left[\frac{(-1+p) p^{3}}{H^{2}+\left(H^{2}+\dot{H}\right)}\right]^{-\frac{3}{4} p(1+\omega)}+4608 H^{4}\left\{-\left((1+\omega)\left[-2+\frac{3}{4} p(1+\omega)\right]\right.\right. \\
& \left.\times\left[\frac{H^{2}+\left(H^{2}+\dot{H}\right)}{(-1+p) p^{3}}\right]^{\frac{3}{4}(-4+p+p \omega)}\right]\left[18432(-1+p)^{2} p^{8}(-1+p(4+3 \omega))\right]^{-1} \\
& \left.-p\left[6144(1+p) H^{2}\left(H^{2}+\dot{H}\right)^{2} \sqrt{\frac{p H^{2}\left(H^{2}+\dot{H}\right)}{-1+p}}\right]^{-1}\right\}\left[2 \dot{H}^{2}\left(2 H^{2}+\dot{H}\right)+H \ddot{H}\right]^{2} \\
& +\left\{p \left[[2-2 p(4+3 \omega)] H^{4}-2[-1+p(4+3 \omega)] H^{2} \dot{H}+\left(-1+p^{2}\right)(1+\omega)\right.\right. \\
& \times\left[\frac{H^{2}\left(H^{2}+\dot{H}\right)}{(-1+p) p^{3}}\right]^{\frac{3}{4} p(1+\omega)} \sqrt{\frac{p H^{2}\left(H^{2}+\dot{H}\right)}{-1+p}}\left[\left[4 H^{4} \dot{H}-6 \dot{H}^{3} \ddot{H}-8 H \dot{H} \ddot{H}-H^{2}\left(18 \dot{H}^{2}\right.\right.\right. \\
& \left.\left.\left.+H^{3}\right)\right]\right\}\left[4(1+P)[-1+p(4+3 \omega)] H^{2}\left(H^{2}+\dot{H}\right)^{2} \sqrt{\frac{p H^{2}\left(H^{2}+\dot{H}\right)}{-1+p}}\right]^{-1}=0
\end{aligned}
$$

The Equation (57) is a differential equation in $H(t)$ which admits for solution $H(t)=\frac{p}{t}$. Thus, from relations (50)-(52), we obtain:

$$
\begin{aligned}
\varepsilon_{1} & =\frac{1}{p} \\
\varepsilon_{2} & =0 \\
\varepsilon_{3} & =\frac{1}{p}
\end{aligned}
$$

then, the relations (53)-(56) lead to:

$$
\begin{gathered}
r \approx \frac{16}{p} \\
n_{s} \approx 1-\frac{2}{p} \\
\alpha_{s} \approx 0 \\
n_{T} \approx-\frac{2}{p}
\end{gathered}
$$

These are the values of the observables of power law inflation. We will now de- 
termine a $F(G)$ model capable of describing such inflation and which is in agreement with Planck's results.

According to Planck's results, we have: $0.962 \leq n_{s} \leq 0.974$ and by virtue of equality (62), we obtain: $52.632 \leq p \leq 76.923$.

By posing for example $p=60$, we obtain: $r=0.266, n_{s}=0.966, \alpha_{s}=0$ and $n_{T}=-0.033$ which are values actually in agreement with Planck's results. Considering that just after inflation, the universe is essentially dominated by radiation, we have $\omega=1 / 3$ and the model becomes:

$$
F(G)=C_{2} G^{\frac{-59}{4}}-\frac{3 \sqrt{590}}{61} \sqrt{G}+C_{1} G-\frac{\rho_{0}}{4.042 .10^{511}} G^{60}
$$

Note that it is possible to derive a $F(G)$ model family describing inflation and in perfect agreement with Planck's results by suitably choosing the values of the parameter $p$ of our model.

The question that is actually to be asked consists in checking if the inflationary model (65) extols the exit from inflation.

\subsection{Graceful Exit from Inflation}

Several approaches are used to study the exit from inflation. Based on the dynamic of the Hubble parameter, the exit from inflation are succesfully performed through the type VI singularity survey [45] and through the dynamic of the slow-roll parameters [46] [47]. For the first time, the authors in [48] have performed the exit from inflation by involving quantum effects coming from trace anomaly equation and giving a de Sitter solution whose instability scores the graceful exit from inflation. In [49], from an autonomous dynamical system reconstructed from $f(R)$ motion equation and assisted by scalar field, they numerically provide a de Sitter attractor which becomes unstable for e.fold number $N=60$ (end of inflation), proof of a graceful exit from the inflationary era. The actor of this exit is the scalar field because in their previous analysis [50] without scalar field, the obtained de Sitter solution is eternally stable. This last result is confirmed in $f(T)$ theory by [51]. On can conclude from these last approach that the instability in a dynamical evolution is a source of graceful exit.

Recently, the authors in [9] have analysed an intrinsic dynamic in $f(G)$ theory where some conditions for the cosmological viability of $f(G)$ dark energy models have been performed. Through an approach based on autonomous dynamical system depending from two single parameters $m$ and $r$, they provide natural conditions in the $f(G)$ gravity to describe a ordinary matter or radiation epoch, the dark energy epoch and the transition between the both epochs. The fundamental required condition is that the $m(r)$ curve should pass through $(-1 / 2 ;-1 / 2)$ in the $m-r$ plane. This means the same point $(-1 / 2,-1 / 2)$ corresponds to ordinary matter or radiation domination and the dark energy domination. So the critical points manifesting this property must be unstable in order to pass from matter or radiation domination epoch to the dark matter domination epoch. Such instability is reached when the parametric function 

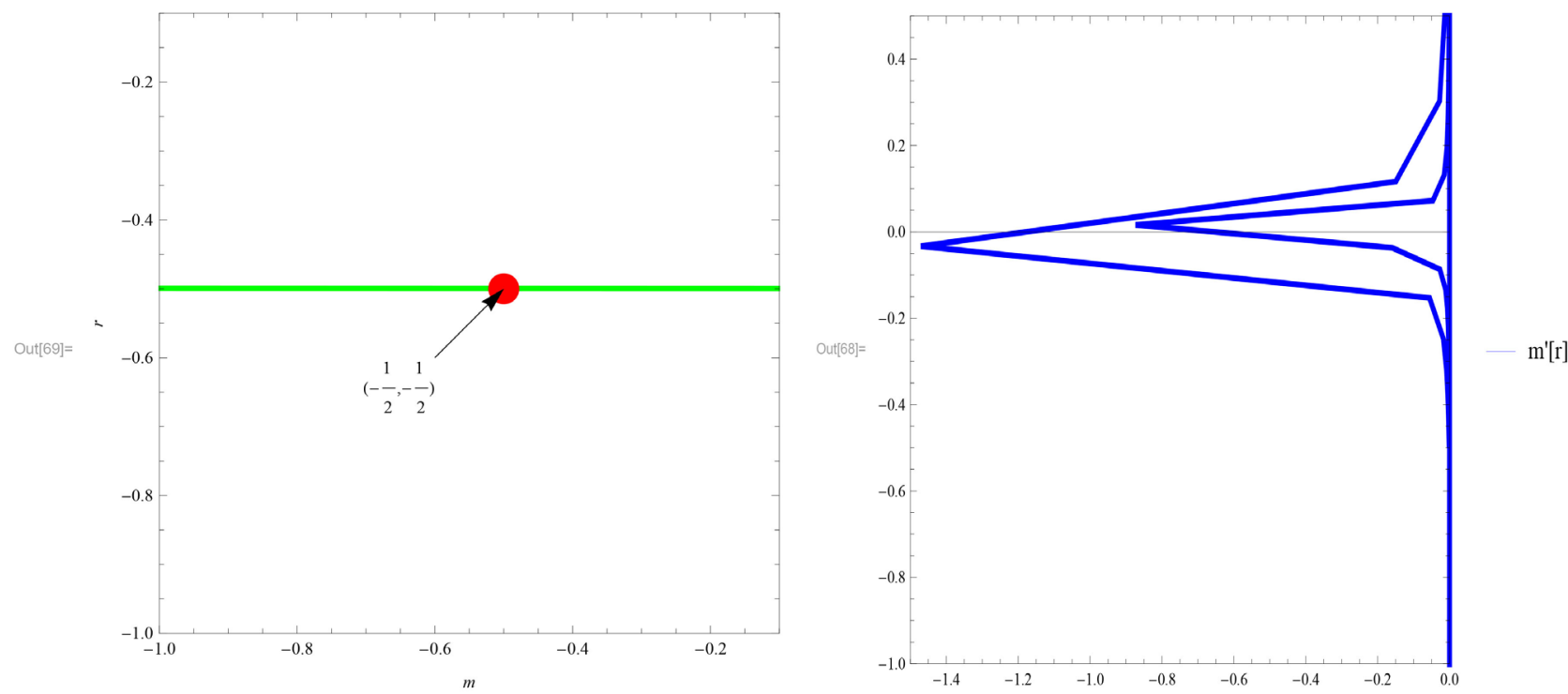

Figure 3. Evolution of $m(r)$ (green curve at left) and its first derivative $m^{\prime}(r)$ (blue curve at right) for model (65) with $\rho_{0}=10^{200}, C_{1}=1.39727 \times 10^{45}$ and $C_{2}=1.14346 \times 10^{138}$.

$m(r)$ satisfies the condition $m^{\prime}(-1 / 2)>-1$. The parameter $m$ and $r$ are given by

$$
m(G)=\frac{G f^{\prime \prime}(G)}{f^{\prime}(G)} \text { and } \quad r(G)=-\frac{G f^{\prime}(G)}{f(G)}
$$

without providing the functional form of $m(r)$ and $m^{\prime}(r)$ in the framework of the inflationary model (65), their parametric plot leads to the Figure 3. The green curve shows clearly the key point $(-1 / 2,-1 / 2)$ is reached meaning that this model can truly lead the radiation domination era. The condition of transition from the radiation domination era to ordinary matter domination era or dark energy domination era is clearly appreciable via the blue curve in the same Figure 3 because it shows $m^{\prime}(-1 / 2)>-1$. Such instability is qualified graceful exit from inflation [52].

\section{Conclusion}

During this study, we devoted to the reconstruction of a model in the modified theory of gravity $F(G)$. From well elucidated processes, we have conducted the study of its stability. The results obtained clearly show that the reconstructed model is stable for the both de Sitter and the power-law evolutions. Therefore, this model is potentially suitable for dealing with cosmological questions such as the inflation of the Universe, the explanation of the different phases of the evolution of the Universe, and many others. In this work, the question of the Universe inflation was also approached. The study made it possible to find the observables within the framework of a scale factor evolving according to the power law. This study also made it possible to determine a model describing the inflation of the universe and which is in agreement with Planck's results. Our analy- 
sis shows that the obtained top model develops clearly the graceful exit from the inflation.

\section{Conflicts of Interest}

The authors declare no conflicts of interest regarding the publication of this paper.

\section{References}

[1] Fabris, J. (2004) Introduction à la cosmologie. Ecole Internationale sur les Structures Géométriques et Applications, Dakar.

[2] Riess, G., Filippenko, A.V., Challis, P., et al. (1998) The Astronomical Journal, 116, 1009. https://doi.org/10.1086/300499

[3] Perlmutter, S., Aldering, G., Goldhaber, G., et al. (1998) The Astrophysical Journal, 517,565

[4] Komatsu, E., Dunkley, J., Nolta, M.R., et al. (2008) The Astrophysical Journal Supplement Series, 180, 330.

[5] Komatsu, E., Smith, K.M., Dunkley, J., et al. (2011) The Astrophysical Journal Supplement Series, 192, 18.

[6] Bamba, K., Capozziello, S., Nojiri, S. and Odintsov, S.D. (2012) Astrophysics and Space Science, 342, 155-228. https://doi.org/10.1007/s10509-012-1181-8

[7] Nojiri, S. and Odintsov, S.D. (2005) Physics Letters B, 631, 1.

[8] Cognola, G., Elizalde, E., Nojiri, S., Odintsov, S.D. and Zerbini, S. (2006) Physical Review D, 73, 084007. https://doi.org/10.1103/PhysRevD.73.084007

[9] Zhou, S.Y., Copeland, E.J. and Saffin, P.M. (2009) Journal of Cosmology and Astroparticle Physics, 2009, JCAP07(2009)009.

https://doi.org/10.1088/1475-7516/2009/07/009

[10] Chiba, T. (2005) Journal of Cosmology and Astroparticle Physics, 2005, 8. https://doi.org/10.1088/1475-7516/2005/03/008

[11] Cognola, G., Elizalde, E., Nojiri, S., Odintsov, S.D. and Zerbini, S. (2007) Physical Review D, 75, 086002. https://doi.org/10.1103/PhysRevD.75.086002

[12] De Felice, A., Hindmarsh, M. and Trodden, M. (2006) Journal of Cosmology and Astroparticle Physics, 8, 5. https://doi.org/10.1088/1475-7516/2006/08/005

[13] Amendola, L., Charmousis, C. and Davis, S.C. (2006) Journal of Cosmology and Astroparticle Physics, 12, 020. https://doi.org/10.1088/1475-7516/2006/12/020

[14] Motaharfara, M. and Sepangi, H.R. (2016) The European Physical Journal C, 76, Article No. 646. https://doi.org/10.1140/epjc/s10052-016-4474-1

[15] Koivisto, T. and Mota, D. (2006) Physics Letters B, 644, 104-108. https://doi.org/10.1016/j.physletb.2006.11.048

[16] Sanyal, A.K. (2007) Physics Letters B, 645, 1-5. https://doi.org/10.1016/j.physletb.2006.11.070

[17] Uddin, K., Lidsey, J.E. and Tavakol, R. (2009) General Relativity and Gravitation, 41, No. 12.

[18] Houndjo, S., Rodrigues, M., Momeni, D. and Myrzakulov, R. (2013) Canadian Journal of Physics, 92, 1528-1540.

[19] Rodrigues, M.E., Houndjo, M.J.S., Momeni, D. and Myrzakulov, R. (2013) Canadian Journal of Physics, 92, No. 2. https://doi.org/10.1139/cjp-2013-0414 
[20] Nojiri, S., Odintsov, S., Toporensky, A. and Tretyakov, P. (2010) General Relativity and Gravitation, 42, 1997-2008.

[21] Setare, M.R. and Mohammadipour, N. (2012) Cosmography in $F(G)$ Modified Gravity. ar $\chi$ iv:1206.0245v1 [physics.gen-ph].

[22] Li, B., Barrow, J.D. and Mota, D.F. (2007) Physical Review D, 76, 044027. https://doi.org/10.1103/PhysRevD.76.044027

[23] Boehmer, C.G. and Lobo, F.S.N. (2009) Physical Review D, 79, 067504.

[24] Guth, A.H. (1981) Physical Review D, 23, 347. https://doi.org/10.1103/PhysRevD.23.347

[25] Linde, A.D. (1983) Physics Letters B, 129, 177-181. https://doi.org/10.1016/0370-2693(83)90837-7

[26] Bamba, K., Nojiri, S., Odintsov, S.D. and Sáez-Gómez, D. (2014) Physical Review D, 90, 124061.

[27] Bamba, K., Nojiri, S. and Odintsov, S.D. (2014) Physics Letters B, 737, 374-378. https://doi.org/10.1016/j.physletb.2014.09.014

[28] Gao, Q. and Gong, Y. (2014) Physics Letters B, 734, 41-43. https://doi.org/10.1016/j.physletb.2014.05.018

[29] Odintsov, S.D. and Oikonomou, V.K. (2018) Physical Review D, 98, ID: 044039.

[30] Kleidis, K. and Oikonomou, V.K. (2018) International Journal of Geometric Methods in Modern Physics, 15, 1850137. https://doi.org/10.1142/S0219887818501372

[31] Kleidis, K. and Oikonomou, V.K. (2018) International Journal of Geometric Methods in Modern Physics, 15, 1850212. https://doi.org/10.1142/S0219887818502122

[32] Odintsov, S.D. and Oikonomou, V.K. (2018) Physical Review D, 98, 024013. https://doi.org/10.1103/PhysRevD.98.024013

[33] Odintsov, S.D. and Oikonomou, V.K. (2015) Big-Bounce with Finite-time Singularity: The $F(R)$ Gravity Description. arXiv:1512.04787 [gr-qc].

[34] Odintsov, S.D., Oikonomou, V.K., Fronimos, F.P. and Fasoulakos, K.V. (2010) Unification of a Bounce with a Viable Dark Energy Era in Gauss-Bonnet Gravity. arXiv:2010.13580.

[35] Houndjo, M.J.S. (2017) The European Physical Journal C, 77, Article No. 607. https://doi.org/10.1140/epjc/s10052-017-5171-4

[36] Rudra, P. (2014) International Journal of Modern Physics D, 24, No. 2.

[37] Nojiri, S. and Odintsov, S. (2005) Physics Letters B, 631, 1-6. https://doi.org/10.1016/j.physletb.2005.10.010

[38] Nojiri, S., Odintsov, S.D. and Oikonomou, V.K. (2017) Physics Reports, 692, 1-104. https://doi.org/10.1016/j.physrep.2017.06.001

[39] Bamba, K., Odintsov, S. and Saridakis, E. (2017) Modern Physics Letters A, 32, Article ID: 1750114

[40] Houndjo, S. (2017) The European Physical Journal C, 77, 607.

[41] Goheer, N., Goswami, R., Larena, J., Dunsby, P.K.S. and Ananda, K. (2009) AIP Conference Proceedings, 1241, 898. https://doi.org/10.1063/1.3462731

[42] De Felice, A. and Tsujikawa, S. (2009) Physics Letters B, 675, 1-8.

[43] de la Cruz-Dombriz, A. and Sáez-Gómez, D. (2012) Classical and Quantum Gravity, 29, 245014.

[44] Cai, Y., Capozziello, S., De Laurentis, M. and Saridakis, E. (2015) Reports on Progress in Physics, 79, 106901. 
[45] Odinstov, S.D. and Oikonomou, V.K. (2015) Singular Inflationary Universe from $F(R)$ Gravity. arXiv: 1510.04333 [gr-qc].

[46] Odintsov, S.D. and Oikonomou, V.K. (2015) Physical Review D, 92, 024058.

[47] Odintsov, S.D. and Oikonomou, V.K. (2015) Physical Review D, 92, 124024. https://doi.org/10.1103/PhysRevD.92.124024

[48] Bamba, K., Nojiri, S. and Odintsov, S.D. (2014) Physics Letters B, 731, arXiv: 1401. 7378v1[gr-qc].

[49] Kleidis, K. and Oikonomou, V.K. (2018) Autonomous Dynamical System Description of de Sitter Evolution in Scalar Assisted $f(R)-\phi$ Gravity. [arXiv:1808.04674 [gr$\mathrm{qc}]$ ].

[50] Odintsov, S.D and Oikonomou, V.K. (2017) Physical Review D, 96, Article: 104049. [arXiv:1711.02230v1[gr-qc]]. https://doi.org/10.1103/PhysRevD.96.104049

[51] Ganiou, M.G., Logbo, P.H., Houndjo, M.J. and Tossa, J. (2019) The European Physical Journal Plus, 134, Article No. 45. https://doi.org/10.1140/epjp/i2019-12393-8

[52] Nashed, G.G.L., El Hanafy, W. and Ibrahim, Sh.Kh. (2014) Graceful Exit from $f(T)$ Gravity. arXiv:1411.3293[gr-qc]. 